\title{
Spectral and Polar Decomposition in AW*-Algebras
}

\author{
M. Frank
}

We show the possibility and the uniqueness of polar decomposition of elements of arbitrary $\mathrm{AW}^{*}$-algebras inside them. We prove that spectral decomposition of normal elements of normal $\mathrm{AW}^{*}$-algebras is possible and unique inside them. The possibility of spectral decomposition of normal elements does not depend on the normality of the $\mathrm{AW}^{*}$-algebra under consideration.

Key words: Operator algebras, monotone complete $C^{*}$-algebras, $A W^{*}$-algebras, spectral decomposition and polar decomposition of operators

AMS subject classification: 46L05, 46L35, 47C15

The spectral decomposition of normal linear (bounded) operators and the polar decomposition of arbitrary linear (bounded) operators on Hilbert spaces have been interesting and technically useful results in operator theory [3, 9, 13, 20]. The development of the concept of von Neumann algebras on Hilbert spaces has shown that both these decompositions are always possible inside of the appropriate von Neumann algebra [14. New light on these assertions was shed identifying the class of von Neumann algebras among all $\mathrm{C}^{*}$-algebras by the class of $\mathrm{C}^{*}$-algebras which possess a pre-dual Banach space, the $\mathrm{W}^{*}$ algebras. The possibility of $\mathrm{C}^{*}$-theoretical representationless descriptions of spectral and polar decomposition of elements of von Neumann algebras (and may be of more general $\mathrm{C}^{*}$-algebras) inside them has been opened up. Steps to get results in this direction were made by several authors. The $\mathrm{W}^{*}$-case was investigated by S. Sakai in 1958-60, [18, [19]. Later on J. D. M. Wright has considered spectral decomposition of normal elements of embeddable $\mathrm{AW}^{*}$-algebras, i.e., of $\mathrm{AW}^{*}$-algebras possessing a faithful von Neumann type representation on a self-dual Hilbert $\mathbf{A}$-module over a commutative $\mathrm{AW}^{*}$-algebra $\mathbf{A}$ (on a so called Kaplansky-Hilbert module), 23, 24]. But, unfortunately, not all $\mathrm{AW}^{*}$-algebras are embeddable. In 1970 J. Dyer [5] and O. Takenouchi [21] gave (*-isomorphic) examples of type III, non-W*, AW*-factors, (see also K. Saitô 15]). Polar decomposition inside $\mathrm{AW}^{*}$-algebras was considered by I. Kaplansky [12] in 1968 and by S. K. Berberian [3] in 1972. They have shown the possibility of polar decomposition in several types of AW*_ algebras, but they did not get a complete answer. In the present paper the partial result of I. Kaplansky is used that $\mathrm{AW}^{*}$-algebras without direct commutative summands and with a decomposition property for it's elements like described at Corollary 5 below allow polar decomposition inside them, [3, §21: Exerc. 1] and [12, Th. 65]. For a detailled overview on these results we refer to [3].

The aim of the present paper is to show that both these decompositions are possible inside arbitrary $\mathrm{AW}^{*}$-algebras without additional assumptions to their structures. 
Recall that an $\mathrm{AW}^{*}$-algebra is a $\mathrm{C}^{*}$-algebra for which the following two conditions are satisfied (cf. I. Kaplansky [10]):

(a) In the partially ordered set of projections every set of pairwise orthogonal projections has a least upper bound.

(b) Every maximal commutative $*$-subalgebra is generated by its projections, i.e., it is equal to the smallest closed $*$-subalgebra containing its projections.

An $\mathrm{AW}^{*}$-algebra $\mathbf{A}$ is called to be monotone complete if every increasingly directed, norm-bounded net of self-adjoint elements of $\mathbf{A}$ possesses a least upper bound in $\mathbf{A}$. An $\mathrm{AW}^{*}$-algebra $\mathbf{A}$ is called to be normal if the supremum of every increasingly directed net of projections of $\mathbf{A}$ being calculated with respect to the set of all projections of $\mathbf{A}$ is it's supremum with respect to the set of all self-adjoint elements of $\mathbf{A}$ at once, cf. [25, [16]. For the most powerful results on these problems see [4] and [17].

To formulate the two theorems the following definitions are useful.

Definition 1 (J. D. M. Wright [24, p.264]): A measure $m$ on a compact Hausdorff space $X$ being valued in the self-adjoint part of a monotone complete $A W^{*}$-algebra is called to be quasi-regular if and only if

$$
m(K)=\inf \{m(U): U-\text { open sets in } X, K \subseteq U\}
$$

for every closed set $K \subseteq X$. We remark that this condition is equivalent to the condition:

$$
m(U)=\sup \{m(K): K-\text { closed sets in } X, K \subseteq U\} \text { for every open set } U \subseteq X \text {. }
$$

Further, if $m(E)=\inf \{m(U): U$-open sets in $X, E \subseteq U\}$ for every Borel set $E \subseteq X$, then the measure $m$ is called to be regular.

Definition 2 (M. Hamana 8, p.260] (cf. [1], [22])): A net $\left\{a_{\alpha}: \alpha \in I\right\}$ of elements of $\mathbf{A}$ converges to an element $a \in \mathbf{A}$ in order if and only if there are bounded nets $\left\{a_{\alpha}^{(k)}: \alpha \in I\right\}$ and $\left\{b_{\alpha}^{(k)}: \alpha \in I\right\}$ of self-adjoint elements of $\mathbf{A}$ and self-adjoint elements $a^{(k)} \in \mathbf{A}, k=1,2,3,4$, such that

(i) $0 \leq a_{\alpha}^{(k)}-a^{(k)} \leq b_{\alpha}^{(k)}, k=1,2,3,4, \alpha \in I$,

(ii) $\left\{b_{\alpha}^{(k)}: \alpha \in I\right\}$ is decreasingly directed and has greatest lower bound zero,

(iii) $\sum_{k=1}^{4}(\mathbf{i})^{k} a_{\alpha}^{(k)}=a_{\alpha}$ for every $\alpha \in I, \sum_{k=1}^{4}(\mathbf{i})^{k} a^{(k)}=a($ where $\mathbf{i}=\sqrt{-1})$.

We denote this type of convergence by $\operatorname{LIM}\left\{a_{\alpha}: \alpha \in I\right\}=a$.

By [6, p.260] the order limit of $\left\{a_{\alpha}: \alpha \in I\right\}$ does not depend on the special choice of the nets $\left\{a_{\alpha}^{(k)}: \alpha \in I\right\},\left\{b_{\alpha}^{(k)}: \alpha \in I\right\}$ and of the elements $a^{(k)}, k=1,2,3,4$. If $\mathbf{A}$ is a commutative $A W^{*}$-algebra, then the notion of order convergence defined above is equivalent to the order convergence in $\mathbf{A}$ which was defined by H.Widom [22] earlier. Note that (cf. [8, Lemma 1.2]) if $\operatorname{LIM}\left\{a_{\alpha}: \alpha \in I\right\}=a, \operatorname{LIM}\left\{b_{\beta}: \beta \in J\right\}=b$, then

(i) $\operatorname{LIM}\left\{a_{\alpha}+b_{\beta}: \alpha \in I, \beta \in J\right\}=a+b$,

(ii) $\operatorname{LIM}\left\{x a_{\alpha} y: \alpha \in I\right\}=x a y$ for every $x, y \in A$,

(iii) $\operatorname{LIM}\left\{a_{\alpha} b_{\beta}: \alpha \in I, \beta \in J\right\}=a b$,

(iv) $a_{\alpha} \leq b_{\alpha}$ for every $\alpha \in I=J$ implies $a \leq b$,

(v) $\|a\|_{A} \leq \lim \sup \left\{\left\|a_{\alpha}\right\|_{A}: \alpha \in I\right\}$.

Furthermore, we need the following lemma describing the key idea of the present paper and being of interest on its own. 
Lemma 3 : Let $\mathbf{A}$ be an $A W^{*}$-algebra and $\mathbf{B} \subseteq \mathbf{A}$ be a commutative $C^{*}$-subalgebra. Then the monotone closures $\hat{\mathbf{B}}(\mathbf{D}), \hat{\mathbf{B}}\left(\mathbf{D}^{\prime}\right)$ of $\mathbf{B}$ inside arbitrary two maximal commutative $C^{*}$-subalgebras $\mathbf{D}, \mathbf{D}^{\prime}$ of $\mathbf{A}$ which contain $\mathbf{B}$, respectively, are *-isomorphic commutative $A W^{*}$-algebras. Moreover, all monotone closures $\hat{\mathbf{B}}(\mathbf{D})$ of $\mathbf{B}$ of this type coincide as $C^{*}$ subalgebras of $\mathbf{A}$ if $\mathbf{A}$ is normal.

Proof: Let $\mathbf{D}$ be a maximal commutative $\mathrm{C}^{*}$-subalgebra of $\mathbf{A}$ containing $\mathbf{B}$. By definition $\mathbf{D}$ is generated by its projections. Let $p \in \hat{\mathbf{B}}(\mathbf{D})$ be a projection. Suppose $p \notin \mathbf{B}$. Then $p$ is the supremum of the set $\mathcal{P}=\left\{x \in \mathbf{B}_{h}^{+} \subseteq \mathbf{D}: x \leq p\right\}$ by [7, Lemma 1.7]. In particular, $\left(1_{A}-p\right)$ is the maximal annihilator projection of $\mathcal{P}$ inside $\mathbf{D}$. But, $\mathcal{P}^{2}=\mathcal{P}$ and, hence, the supremum of $\mathcal{P}$ being calculated inside $\mathbf{D}^{\prime}$ is a projection $p^{\prime}$ again, and $\left(1_{A}-p^{\prime}\right)$ is the maximal annihilator projection of $\mathcal{P}$ in $\mathbf{D}^{\prime}$. Changing the possitions of $\mathbf{D}$ and $\mathbf{D}^{\prime}$ one finds a one-to-one correspondence between the projections of $\hat{\mathbf{B}}(\mathbf{D})$ and $\hat{\mathbf{B}}\left(\mathbf{D}^{\prime}\right)$.

Moreover, the product projection $p_{1} p_{2}$ of two projections $p_{1}, p_{2} \in \hat{\mathbf{B}}(\mathbf{D})$ corresponds to the supremum of the intersection set of the two appropriate sets $\mathcal{P}_{1}$ and $\mathcal{P}_{2}$ of elements of $\mathbf{B}$, and hence, to the product projection $p_{1}^{\prime} p_{2}^{\prime}$ of the corresponding two projections $p_{1}^{\prime}, p_{2}^{\prime} \in \hat{\mathbf{B}}\left(\mathbf{D}^{\prime}\right)$. That is, the found one-two-one correspondence between the sets of projections of $\hat{\mathbf{B}}(\mathbf{D})$ and of $\hat{\mathbf{B}}\left(\mathbf{D}^{\prime}\right)$ preserves the lattice properties of these nets. Since $\hat{\mathbf{B}}(\mathbf{D})$ and $\hat{\mathbf{B}}\left(\mathbf{D}^{\prime}\right)$ are commutative $\mathrm{AW}^{*}$-algebras (i.e. both they are linearly spanned by their projection lattices as linear spaces and as Banach lattices), this one-to-one correspondence extends to a $*$-isomorphism of $\hat{\mathbf{B}}(\mathbf{D})$ and $\hat{\mathbf{B}}\left(\mathbf{D}^{\prime}\right)$.

Now, fix such a set $\mathcal{P} \subseteq \mathbf{B}$. By [10] there exists a global maximal annihilator projection $\left(1_{A}-q\right)$ of $\mathcal{P}$ in $\mathbf{A}$. The problem arrising in this situation can be formulated as follows: Does $q$ commute with $\mathbf{B}$, i.e. is $q$ an element of $\mathbf{D}$ and, hence, of every maximal commutative $\mathbf{C}^{*}$-subalgebra $\mathbf{D}^{\prime}$ of $\mathbf{A}$ containing $\mathbf{B}$ ? Obviously, $\left(1_{A}-q\right)$ is the supremum of the set of all those annihilator projections $\left\{\left(1_{A}-p\right)\right\}$ which we have constructed above, but only in the sense of a supremum in the net of all projections of $\mathbf{A}$ since monotone completeness or normality of $\mathbf{A}$ are not supposed, in general. So we have to assume that A is normal, cf. [25, 16], to be sure in our subsequent conclusions. Then there follows that $\left(1_{A}-q\right)$ has to be the supremum of the set of the set of all those projections $\left\{\left(1_{A}-p\right)\right\}$ in the self-adjoint part of $\mathbf{A}$. Hence, $q$ commutes with $\mathbf{B}$ since each of the projections $p$ do. This means that $q$ belongs to every maximal commutative $\mathrm{C}^{*}$-subalgebra $\mathbf{D}$ of $\mathbf{A}$ containing $\mathbf{B}$ because of their maximality, and $q=p$ for every $p \in \mathbf{D}$ since $q \leq p$, and $p$ was the supremum of $\mathcal{P}$ inside $\mathbf{D}_{h}^{+}$.

Since $\mathcal{P}$ was fixed arbitrarily one concludes that $\hat{\mathbf{B}}(\mathbf{D})$ does not depend on the choice of $\mathbf{D}$ inside normal $\mathrm{AW}^{*}$-algebras $\mathbf{A}$.

Theorem 4 (cf. [24, Th.3.1 and Th.3.2]): Let $\mathbf{A}$ be a normal $A W^{*}$-algebra and $a \in \mathbf{A}$ be a normal element. Let $\mathbf{B} \subseteq \mathbf{A}$ be that commutative $C^{*}$-subalgebra in $\mathbf{A}$ being generated by the elements $\left\{1_{A}, a, a^{*}\right\}$, and denote by $\hat{\mathbf{B}}$ the smallest commutative $A W^{*}$-algebra inside $\mathbf{A}$ containing $\mathbf{B}$ and being monotone complete inside every maximal commutative $C^{*}$-subalgebra of $\mathbf{A}$. Then there exists a unique quasi-regular $\hat{\mathbf{B}}$-valued measure $m$ on the spectrum $\sigma(a) \subset \mathbf{C}$ of $a \in \mathbf{A}$, the values of which are projections in $\hat{\mathbf{B}}$ and for which the integral

$$
\int_{\sigma(a)} \lambda d m_{\lambda}=a
$$

exists in the sense of order convergence in $\hat{\mathbf{B}} \subseteq \mathbf{A}$. 
If $\mathbf{A}$ is not normal, then for every maximal commutative $C^{*}$-subalgebra $\mathbf{D}$ of $\mathbf{A}$ containing $\mathbf{B}$ there exists a unique spectral decomposition of $a \in \mathbf{A}$ inside the monotone closure $\hat{\mathbf{B}}(\mathbf{D})$ of $\mathbf{B}$ with respect to $\mathbf{D}$. But, it is unique only in the sense of the *-isomorphy of $\hat{\mathbf{B}}(\mathbf{D})$ and $\hat{\mathbf{B}}\left(\mathbf{D}^{\prime}\right)$ for every two different maximal commutative $C^{*}$-subalgebras $\mathbf{D}, \mathbf{D}^{\prime}$ of $\mathbf{A}$ containing $\mathbf{B}$.

If $\mathbf{A}$ is a $W^{*}$-algebra, then $m$ is regular and the integral exists in the sense of norm convergence.

Proof: By the Gelfand-Naimark representation theorem the commutative $\mathrm{C}^{*}$-subalgebra $\mathbf{B} \subseteq \mathbf{A}$ being generated by the elements $\left\{1_{A}, a, a^{*}\right\}$ is $*$-isomorphic to the commutative $\mathrm{C}^{*}$-algebra $C(\sigma(a))$ of all complex-valued continuous functions on the spectrum $\sigma(a) \subset \mathbf{C}$ of $a \in \mathbf{A}$. Denote this $*$-isomorphism by $\phi, \phi: C(\sigma(a)) \longrightarrow \mathbf{B}$. The isomorphism $\phi$ is isometric and preserves order relations between self-adjoint elements and, hence, positivity of self-adjoint elements. Therefore, $\phi$ is a positive mapping.

Selecting an arbitrary maximal abelian $\mathrm{C}^{*}$-subalgebra $\mathbf{D}$ of $\mathbf{A}$ containing $\mathbf{B}$ one can complete $\mathbf{B}$ to $\hat{\mathbf{B}}(\mathbf{D})$ with respect to the order convergence in $\mathbf{D}$. Note that $\hat{\mathbf{B}}(\mathbf{D}) \subseteq \mathbf{A}$ does not depend on the choice of $\mathbf{D}$ by the previous lemma if $\mathbf{A}$ is normal.

Now, by [24], 23, Th.4.1] there exists a unique positive quasi-regular $\hat{\mathbf{B}}(\mathbf{D})$-valued measure $m$ with the property that

$$
\int_{\sigma(a)} f(\lambda) d m_{\lambda}=\phi(f)
$$

for every $f \in C(\sigma(a))$. Since $\phi^{-1}(a)(\lambda)=\lambda$ for every $\lambda \in \sigma(a) \subset \mathbf{C}$ by the definition of $\phi$ one gets

$$
\int_{\sigma(a)} \lambda d m_{\lambda}=a
$$

Moreover, since the extension $\hat{\phi}$ of $\phi$ to the set of all bounded Borel functions on $\sigma(a)$ fulfils $\hat{\phi}\left(\chi_{E}\right)^{2}=\hat{\phi}\left(\chi_{E}^{2}\right)=\hat{\phi}\left(\chi_{E}\right)$ for the characteristic function $\chi_{E}$ of every Borel set $E \in \sigma(a)$ the measure $m$ is projection-valued, cf. [24]. One finishes refering to Lemma 3

The following corollary is essential to get the polar decomposition theorem.

Corollary 5 : Let $\mathbf{A}$ be an $A W^{*}$-algebra and $x \in \mathbf{A}$ be different from zero. Then there exists a projection $p \in \mathbf{A}_{h}^{+}, p \neq 0$, and an element $a \in \mathbf{A}_{h}^{+}$such that $a, p$ and $\left(x x^{*}\right)^{1 / 2}$ commute pairwise, and $a\left(x x^{*}\right)^{1 / 2}=\left(a x x^{*} a\right)^{1 / 2}=p$.

Proof: Consider the commutative $\mathrm{C}^{*}$-subalgebra $\mathbf{B}$ of $\mathbf{A}$ being generated by the elements $\left\{1_{A}, x x^{*}\right\}$. By the spectral theorem there exists a unique positive quasi-regular measure $m$ on the Borel sets of $\sigma\left(\left(x x^{*}\right)^{1 / 2}\right) \subset \mathbf{R}^{+}$being projection-valued in the monotone closure $\hat{\mathbf{B}}(\mathbf{D}) \subseteq \mathbf{A}$ of $\mathbf{B}$ with respect to an arbitrarily chosen, but fixed, maximal commutative $\mathbf{C}^{*}$-subalgebra $\mathbf{D}$ of $\mathbf{A}$ containing $\mathbf{B}$, and satisfying the equality

$$
\int_{\sigma\left(\left(x x^{*}\right)^{1 / 2}\right)} \lambda d m_{\lambda}=\left(x x^{*}\right)^{1 / 2}
$$

in the sense of order convergence in $\hat{\mathbf{B}}(\mathbf{D}) \subseteq \mathbf{A}$. Now, if $\left(x x^{*}\right)^{1 / 2}$ is a projection, then set $a=1_{A}, p=x x^{*}$. If $\left(x x^{*}\right)^{1 / 2}$ is invertible in $\mathbf{A}$, then set $p=1_{A}, a=\left(x x^{*}\right)^{-1 / 2}$. Otherwise consider a number $\mu \in \sigma\left(\left(x x^{*}\right)^{1 / 2}\right), 0<\mu<\|x\|$, and set $K=[0, \mu] \cap \sigma\left(\left(x x^{*}\right)^{1 / 2}\right)$. The 
value $m(K) \in \hat{\mathbf{B}}(\mathbf{D})$ is a projection different from zero. It commutes with every spectral projection of $\left(x x^{*}\right)^{1 / 2}$ and with $\left(x x^{*}\right)^{1 / 2}$ itself. Since $m$ is a quasi-regular measure one has

$$
\int_{\sigma\left(\left(x x^{*}\right)^{1 / 2}\right) \backslash K} \lambda d\left(m_{\lambda}\left(1_{A}-m(K)\right)\right)=\left(1_{A}-m(K)\right)\left(x x^{*}\right)^{1 / 2} .
$$

Therefore, one finds $p=\left(1_{A}-m(K)\right)$ and $a=\left(\left(1_{A}-m(K)\right)\left(x x^{*}\right)\right)^{-1 / 2}$, where the inverse is taken inside the $\mathrm{C}^{*}$-subalgebra $\left(1_{A}-m(K)\right) \hat{\mathbf{B}}(\mathbf{D}) \subseteq \mathbf{A}$. Since $\mu<\|x\|$ the projection $\mathrm{p}$ is different from zero. The existence of $a \in \mathbf{A}_{h}^{+}$is guaranteed by $0<\mu$

Now we go on to show the polar decomposition theorem for $\mathrm{AW}^{*}$-algebras using results of S. K. Berberian and I. Kaplansky. Previously we need it for commutative $\mathrm{AW}^{*}$-algebras. Note that the proof of the following lemma works equally well for all monotone complete $\mathrm{C}^{*}$-algebras.

Lemma 6 : Let $\mathbf{A}$ be a commutative $A W^{*}$-algebra. For every $x \in \mathbf{A}$ there exists a unique partial isometry $u \in \mathbf{A}$ such that $x=\left(x x^{*}\right)^{1 / 2} u$ and $u u^{*}$ is the range projection of $\left(x x^{*}\right)^{1 / 2}$.

Proof: Throughout the proof we use freely the order convergence inside monotone complete $\mathrm{C}^{*}$-algebras as defined at Definition 2.

First, suppose $x$ to be self-adjoint. The sequence $\left\{u_{n}=x(1 / n+|x|)^{-1}: n \in \mathbf{N}\right\}$ is bounded in norm by the representation theory. It consists of self-adjoint elements, and the sequences $\left\{|x|(1 / n+|x|)^{-1}\right\}$ and $\left\{(|x|-x)(1 / n+|x|)^{-1}\right\}$ are monotone increasing. Hence, the sequence $\left\{u_{n}\right\}$ is order converging inside $\mathbf{A}$, LIM $u_{n}=u$, and $u \in \mathbf{A}_{h}$. Furthermore, the sequence $\left\{u_{n}|x|: n \in \mathbf{N}\right\}$ converges to $x$ in order, i.e., $x=u|x|$. From the equality $x^{2}=|x| u^{*} u|x|$ one draws $u^{*} u \geq \operatorname{rp}(|x|)$ (where $\operatorname{rp}(|x|)$ denotes the range projection of $|x|$ being an element of $\mathbf{A})$. Hence, $u^{*} u=r p(|x|)$ by construction and $u$ is a partial isometry.

Now suppose $x \in \mathbf{A}$ to be arbitrarily chosen. Consider again the sequence $\left\{u_{n}\right\}$ of elements of $\mathbf{A}$ as defined at the beginning. One has to show the fundamentality of it with respect to the order convergence. Since $\mathbf{A}$ is monotone complete the existence of it's order limit $u$ inside $\mathbf{A}$ will be guaranteed in this case. For the self-adjoint part of the elements of $\left\{u_{n}\right\}$ the inequality

$$
\begin{aligned}
0 \leq & {\left[x\left((1 / n+|x|)^{-1}-(1 / m+|x|)^{-1}\right)+\left((1 / n+|x|)^{-1}-(1 / m+|x|)^{-1}\right) x^{*}\right]^{2} } \\
\leq & 2\left[x\left((1 / n+|x|)^{-1}-(1 / m+|x|)^{-1}\right)^{2} x^{*}+\right. \\
& \left.+\left((1 / n+|x|)^{-1}-(1 / m+|x|)^{-1}\right) x^{*} x\left((1 / n+|x|)^{-1}-(1 / m+|x|)^{-1}\right)\right]
\end{aligned}
$$

is valid for every $n, m \in \mathbf{N}$. The expression of the right side converges weakly to zero as $n, m$ go to infinity in each faithful *-representation of $\mathbf{A}$ on Hilbert spaces. Therefore, it is bounded in norm and converges in order to zero as $n, m$ go to infinity because of the positivity of the expression. Since taking the square root preserves order relations between positive elements of a $\mathrm{C}^{*}$-algebra and since self-adjoint elements have polar decomposition inside $\mathbf{A}$ the order fundamentality of the sequence $\left\{1 / 2\left(u_{n}+u_{n}^{*}\right): n \in \mathbf{N}\right\}$ turns out. The order convergence of the anti-self-adjoint part of the sequence $\left\{u_{n}\right\},\left\{1 / 2 i \cdot\left(u_{n}-u_{n}^{*}\right)\right\}$, can be shown in an analogous way. Hence, there exists LIM $u_{n}=u$ inside $\mathbf{A}$.

Now, from the existence of $\operatorname{LIM} u_{n}|x|=x$ one derives the equality $x=u|x|$. The equality $x^{*} x=|x| u^{*} u|x|$ shows that $u^{*} u \geq \operatorname{rp}(|x|)$ and, consequently, $u^{*} u=r p(|x|)$ by construction, i.e., $u$ is a partial isometry.

To show the uniqueness of polar decomposition inside $\mathbf{A}$ suppose $x=v|x|$ for a partial isometry $v$ with $v^{*} v=r p(|x|)$. Then $v|x|=u|x|$, i.e., $v=v \cdot r p(|x|)=u$ 
Theorem 7 : Let $\mathbf{A}$ be an $A W^{*}$-algebra. For every $x \in \mathbf{A}$ there exists a unique partial isometry $u \in \mathbf{A}$ such that $x=\left(x x^{*}\right)^{1 / 2} u$ and $u u^{*}$ is the range projection of $\left(x x^{*}\right)^{1 / 2}$.

Proof: By [12, Th. 65] polar decomposition is possible inside of all $\mathrm{AW}^{*}$-algebras without direct commutative summands under the supposition that every element of it has the property of Corollary 5, (see also [3, §21: Exerc. 1]). Since polar decomposition works separately in every direct summand we have only to compare Corollary 5 , Lemma 6 and the result of I. Kaplansky

The result of Theorem 7 is of interest also because monotone completeness was not necessary to show it, what is a little bit surprising.

\section{REFERENCES}

[1] Azarnia, N.: Dense operators on a KH-module. Rend. Circ. Mat. Palermo (2) 34 (1985), $105-110$.

[2] Berberian, S. K.: Baer *-rings. Berlin - Heidelberg - New York: Springer-Verlag 1972.

[3] Berberian, S.K.: Notes on spectral theory (Van Nostrand Mathematical Studies: Vol. 5). Princeton: D. van Nostrand Company, Inc. 1966.

[4] Christensen, E. and G. K. Pedersen: Properly infinite $A W^{*}$-algebras are monotone sequentially complete. Bull. London Math. Soc. 16 (1984), 407-410.

[5] Dyer, J.: Concerning AW*-algebras. Notices Amer. Math. Soc. 17 (1970), 788.

[6] Hamana, M.: Regular embeddings of $C^{*}$-algebras in monotone complete $C^{*}$-algebras. J. Math. Soc. Japan 33 (1981), 159 - 183.

[7] Hamana, M.: The centre of the regular monotone completion of a $C^{*}$-algebra. J. London Math. Soc. (2) 26 (1982), 522 - 530.

[8] Hamana, M.: Tensor products for monotone complete $C^{*}$-algebras. Japan. J. Math. 8 (1982), $259-283$.

[9] Hilbert, D.: Grundzüge einer allgemeinen Theorie der linearen Integralgleichungen (Fortschritte der mathematischen Wissenschaften in Monographien: Vol. 3). Leipzig-Berlin: Teubner-Verlag, 1912.

[10] Kaplansky, I.: Projections in Banach algebras. Ann. Math. 53 (1951), 235 - 249.

[11] Kaplansky, I.: Modules over operator algebras. Amer. J. Math. 75 (1953), 839 - 858.

[12] Kaplansky, I.: Rings of operators. New York: Benjamin 1968.

[13] Lengyel, B. A. and M.H. Stone: Elementary proof of the spectral theorem. Ann. Math. 37 (1936), 853 - 864.

[14] Murray, F. J. and J. von Neumann: On rings of operators. Ann. of Math. (2) 37 (1936), $116-229$.

[15] SAitô, K.: $A W^{*}$-algebras with monotone convergence property and type III, non- $W^{*}$, $A W^{*}$-factors. In: $\mathrm{C}^{*}$-algebras and Applications to Physics. Proc. Second Japan-USA Seminar Los Angeles, April 18-22, 1977 (eds.: H. Araki, R. V. Kadison). Lect. Notes Math. 650 (1978), $131-134$. 
[16] SAitô, K.: On normal AW*-algebras. Tôhoku Math. J. 33 (1981), 567-576.

[17] Saitô, K. and J. D. M. Wright: All $A W^{*}$-factors are normal. J. London Math. Soc. (2) 44 (1991), 143-154.

[18] SAKAI, S.: The theory of $W^{*}$-algebras (Lecture Notes Series). New Haven, USA: Yale University 1962.

[19] SakAI, S. $C^{*}$-algebras and $W^{*}$-algebras (Ergebnisse der Mathematik und ihrer Grenzgebiete: Bd.60). Berlin-Heidelberg-New York: Springer-Verlag 1971.

[20] Sz.-Nagy, B.: Spektraldarstellungen linearer Transformationen des Hilbertschen Raumes (Ergebnisse der Mathematik: Vol. 5, Teil 5). Berlin: Springer-Verlag 1942.

[21] Takenouchi, O.: A non- $W^{*}, A W^{*}$-factor. In: $\mathrm{C}^{*}$-algebras and Applications to Physics. Proc. Second Japan-USA Seminar Los Angeles, April 18-22, 1977 (eds.: H. Araki, R. V. Kadison). Lect. Notes Math. 650 (1978), 135 - 139.

[22] Widom, H.: Embedding in algebras of type I. Duke Math. J. 23 (1956), 309 - 324.

[23] Wright, J. D. M.: Stone-algebra-valued measures and integrals. Proc. London Math. Soc. (3) 19 (1969), 107 - 122.

[24] Wright, J. D. M.: A spectral theorem for normal operators on a Kaplansky-Hilbert module. Proc. London Math. Soc. (3) 19 (1969), 258 - 268.

[25] Wright, J. D. M.: Normal AW*-algebras. Proc. Royal Soc. Edinburgh 85A (1980), 137-141.

Received 18.9.91; in revised version 28.4.92

Dr. Michael Frank

Universität Leipzig

Mathematisches Institut

Augustusplatz 10

D(Ost)-7010 Leipzig

frank@mathematik.uni-leipzig.dbp.de 